\title{
Morphological and molecular characterization of Sarcocystis wenzeli in chickens (Gallus gallus) in China
}

Jing Pan ${ }^{1}$, Chunli Ma ${ }^{1}$, Zhumei Huang ${ }^{1}$, Yulong Ye ${ }^{1}$, Hongxia Zeng ${ }^{1}$, Shuangsheng Deng ${ }^{1}$, Junjie Hu²* and Jianping $\mathrm{TaO}^{3}$

\begin{abstract}
Background: There has been considerable confusion concerning the number and classification of Sarcocystis spp. in chickens. Scarce nucleotide data of Sarcocystis spp. from chickens are provided in GenBank. The study aimed to investigate the morphological and molecular characteristics of Sarcocystis spp. found in chickens in China.
\end{abstract}

Methods: Tissues from 33 chickens were collected in 2019. Sarcocysts were observed using light (LM) and transmission electron microscopy (TEM). Individual sarcocysts from different chickens were selected for DNA extraction, and five loci, 185 rDNA, 285 rDNA, ITS1 region, the mitochondrial cox1 gene and the apicoplastic rpoB gene, were amplified from each sarcocyst, sequenced and analyzed.

Results: Only S. wenzeli was found in 14 of 33 (42.4\%) chickens. Under LM, the sarcocysts were microscopic and exhibited palisade-like villar protrusions measuring 1.5-2.8 $\mathrm{mm}$. Ultrastructurally, the sarcocyst wall contained numerous stubby hill-like villar protrusions. The protrusions included scattered microtubules, which extended from the tips of the protrusions into the ground substance. The five loci were successfully sequenced and the sequences deposited in GenBank. At 18S rDNA, ITS1 and cox1, the most similar sequences in GenBank were those of Sarcocystis sp. obtained from the brains of chickens, i.e. $99.9-100 \%, 98.1-98.5 \%$ and $99.3 \%$ identity, respectively. The five loci (18S rDNA, $28 \mathrm{~S}$ rDNA, ITS1, cox1 and rpoB) showed different levels of interspecific sequence similarity with other closely related species of Sarcocystis (e.g. 99.8\%, 99.0-99.2\%, 89.3-89.7\%, 98.5\%, and 97.5\%, respectively, with S. anasi). Phylogenetic analysis based on four of the loci (18S rDNA, cox1, rpoB and ITS1) revealed that $S$. wenzeli formed an independent clade with Sarcocystis spp. that utilize geese or ducks as intermediate hosts and canines as the known or presumed definitive host.

Conclusions: To our knowledge, the sequences of $28 \mathrm{~S}$ rDNA and $r p o B$ reported here constitute the first records of genetic markers of Sarcocystis spp. in chickens. Based on molecular analysis, S. wenzeli might be responsible for the neurological disease in chickens, and ITS1 and rpoB are more suitable for discriminating it from closely related Sarcocystis spp. Phylogenetic analysis revealed that $S$. wenzeli presents a close relationship with Sarcocystis spp. in geese or ducks.

Keywords: Sarcocystis wenzeli, Gallus gallus, Morphological and molecular characterization

\footnotetext{
*Correspondence: jjhu@ynu.edu.cn

${ }^{2}$ School of Ecology and Environmental Sciences and Yunnan Key

Laboratory for Plateau Mountain Ecology and Restoration of Degraded

Environments, Yunnan University, Kunming 650091, China

Full list of author information is available at the end of the article
}

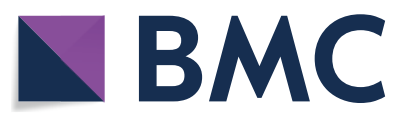

(c) The Author(s) 2020. This article is licensed under a Creative Commons Attribution 4.0 International License, which permits use, sharing, adaptation, distribution and reproduction in any medium or format, as long as you give appropriate credit to the original author(s) and the source, provide a link to the Creative Commons licence, and indicate if changes were made. The images or other third party material in this article are included in the article's Creative Commons licence, unless indicated otherwise in a credit line to the material. If material is not included in the article's Creative Commons licence and your intended use is not permitted by statutory regulation or exceeds the permitted use, you will need to obtain permission directly from the copyright holder. To view a copy of this licence, visit http://creativecommons.org/licenses/by/4.0/. The Creative Commons Public Domain Dedication waiver (http://creativecommons.org/publicdomain/zero/1.0/) applies to the data made available in this article, unless otherwise stated in a credit line to the data. 
Chunli Ma: mc119960108@sohu.com

Yulong Ye: YYL241896@126.com

Hongxia Zeng: Zhx182905@163.com

Junjie Hu: jjhu@ynu.edu.cn

\section{Background}

Species of the genus Sarcocystis exhibit an obligate twohost life-cycle, with sexual development in the small intestine of the definitive host and asexual development in different tissues of the intermediate host, which are usually herbivores. To date, three species of Sarcocystis with chickens (Gallus gallus) as the intermediate host have been named: S. horvathi Rátz, 1908 [1], S. gallinarum Krause and Goranoff [2] and S. wenzeli (Wenzel et al.) Odening 1997 [3, 4]. However, there has been considerable confusion concerning the number and classification of species of Sarcocystis in chickens owing to the imperfection of the original description [5].

The correct identification of Sarcocystis species that might infect chickens is crucial for sarcocystosis control and prevention. The ultrastructure of sarcocysts is traditionally a reliable characteristic for identifying different Sarcocystis species in a given host. Currently, PCR assays and sequencing procedures are considered much more practical, accurate, and reliable methods for the delineation and identification of Sarcocystis species than traditional methods based on morphological characteristics $[6,7]$. However, there are only one $18 S$ rDNA sequence (783 bp), one ITS1 region (ITS1) sequence (923 bp) and one mitochondrial cox 1 gene sequence (547 bp) of Sarcocystis sp. in chickens currently deposited in GenBank. All these nucleotide sequences were obtained from Sarcocystis in chickens associated with neurological lesions in Brazil, and the parasite was closely related to $S$. anasi and $S$. albifronsi [8]. Nevertheless, Sarcocystis sp, found by these authors was not identified to the species level.

Therefore, the aims of the present study were: (i) to obtain data on the prevalence of Sarcocysits in chickens using morphological characteristics; to (ii) sequence and analyze the near-complete $18 S$ rDNA, $28 S$ rDNA, ITS1, cox 1 and apicoplastic rpoB gene (rpoB) of Sarcocystis species found in chickens in order to augment the species descriptions; and (iii) to investigate phylogenetic relationships of Sarcocystis species in chickens with known species of fowl-infecting Sarcocystis spp. using $18 S$ rDNA, $\operatorname{cox} 1, r p o B$ and ITS1 sequences.

\section{Methods}

Morphological examination of sarcocysts from chickens

In total, tissues from 33 chickens were collected from Jiaojiaqing village, Shizong County, Yunnan Province, located in southwestern China, in July and December 2019. These chickens were free ranging and were raised by the local peasants. From each chicken, fresh tissue samples of the skeletal muscles and heart were examined for sarcocysts. In the laboratory, 20 pieces of $3 \mathrm{~mm}$ muscle from each collected sample were pressed and squeezed between two glass slides and inspected using a stereomicroscope. Individual sarcocysts were extracted and isolated from muscle fibers using dissection needles and processed for light (LM) and transmission electron microscopy (TEM) and DNA analysis. For TEM, four sarcocysts (two from chicken no. 4 and two from chicken no. 10$)$ were fixed in $2.5 \%$ glutaraldehyde in cacodylate buffer $(0.1 \mathrm{M}, \mathrm{pH} 7.4)$ at $4{ }^{\circ} \mathrm{C}$ and post-fixed in $1 \%$ osmium tetroxide in the same buffer, then dehydrated in a graded alcohol series and embedded in an epon-alaldite mixture. Ultrathin sections were stained with uranyl acetate and lead citrate and then examined using a JEM100CX transmission electron microscope (JEOL Ltd., Tokyo, Japan) at $80 \mathrm{kV}$. For DNA isolation, individual cysts were stored in sterile water at $-20^{\circ} \mathrm{C}$ prior to processing.

\section{DNA isolation, PCR amplification, cloning and sequence analysis}

For DNA analysis, 5 individual sarcocysts isolated from different chickens were subjected to genomic DNA extraction using the TIANamp Genomic DNA Kit (Tiangen Biotech Ltd., Beijing, China) according to the manufacturer's instructions. The Sarcocystis species were characterized at 5 loci within the $18 S$ rDNA, $28 S$ rDNA, ITS1, cox 1 and rpoB genes. The near-complete $18 S$ rDNA gene was amplified with the primer pair S1/SarDR $[9,10]$; the near-full-length $28 S$ rDNA gene was amplified with the primer sets KL1/KL3, KL4/KL5b, and KL6/KL2 [11]; the complete ITS1 region was amplified with the primer pair P-ITSF/P-ITSR [10]; the partial cox1 gene was amplified with the primer pair SF1/COIRm [6]; and the partial $r p o B$ fragment was amplified with the primer pair rpoBF2 (5'-ATT TTT GTG GAT ATG ATT TTG AAG ATG C-3') and rpoBR2 (5'-AGT TTA GAT CCA GTT CTA CCG-3'), designed using OLIGO 7.60 (Molecular Biology Insights, Inc., West Cascade, USA) based on highly conserved regions of the rpoB sequences of Toxoplasma gondii, Neospora caninum, and Sarcocystis spp. deposited on GenBank. The PCR products were purified, cloned, sequenced, and assembled using the methods described in a previous report [12].

Phylogenetic analyses were conducted separately for the $18 S$ rDNA, cox $1, r p o B$ and ITS1 sequences using 
MEGA X software [13]. The selected sequences of the four loci of Sarcocystis spp. from various hosts were downloaded from GenBank, respectively, and aligned with the ClustalW program integrated in MEGA $\mathrm{X}$ applying a gap opening penalty of $10 / 10$ and a gap extension penalty of $0.1 / 0.2$ as pairwise and multiple alignment parameters, respectively. The alignment was subsequently checked visually; some sequences were truncated at both ends, so all sequences started and ended at the same nucleotide positions. The maximum parsimony (MP) trees were generated with a tree-bisection-regrafting (TBR) algorithm. The reliability of the MP phylograms was tested with the bootstrap method using 1000 replications.

In the case of $18 S \mathrm{rDNA}$, the final alignment comprised a total of 29 nucleotide sequences from 26 taxa and 2100 aligned positions, and Besnoitia besnoiti (GenBank: DQ227418), N. caninum (GenBank: U16159) and T. gondii (GenBank: U03070) were chosen as outgroups to root the tree. At $\operatorname{cox} 1$, the final alignment comprised 28 cox 1 nucleotide sequences from 28 species and 1020 aligned positions with no gaps, and T. gondii (GenBank: JX473253), Hammondia triffittae (GenBank: JX473247) and B. besnoiti (GenBank: XM029362743) were used as outgroup species. At rрoB, the final alignment comprised 20 rpoB nucleotide sequences from 19 species and 694 aligned positions with no gaps, and T. gondii (GenBank: AF095904) and $N$. aninum (GenBank: AF138960) were used as outgroup species to root the tree. At ITS1, the final alignment comprised 25 ITS1 nucleotide sequences from 21 species and 1206 aligned positions, and B. tarandi (GenBank: MH217579) and N. caninum (GenBank: U16159) were chosen as outgroups.

\section{Results}

\section{LM and TEM examination of $S$. wenzeli sarcocysts}

Only sarcocysts resembling those of $S$. wenzeli were found in 14 of $33(42.4 \%)$ chickens. Sarcocysts were found in skeletal muscles but not in the heart. Using LM, the sarcocysts of the parasite were observed to be microscopic, measuring 381-3585 $\times 48-154 \mu \mathrm{m}(n=30)$. The sarcocyst wall exhibited numerous short palisade-like villar protrusions measuring $1.5-2.8 \mu \mathrm{m}(n=40)$ in length (Fig. 1a). The cysts were septate, and their interior compartments were filled with lancet-shaped bradyzoites measuring 9.2-12.6 $\times 1.5-3.5 \mu \mathrm{m}(n=40)$ (Fig. 1b).

Four sarcocysts from both chickens were examined using TEM, all of which appeared to have walls that were ultrastructurally similar and closely resembled the "type 9k" cyst wall. The sarcocyst wall contained numerous stubby hill-like villar protrusions that were up to $1.2 \mu \mathrm{m}$ long and $1.0 \mu \mathrm{m}$ wide and were lined with an electrondense layer that appeared thicker at the tips of the protrusions (Fig. 1c). Within the protrusions, there were numerous scattered fine, electron-dense granules and scattered microtubules. The microtubules extended from the tips of the protrusions into the ground substance, where they crossed microtubules originating from neighboring protrusions (Fig. 1d). The protrusions were spaced at intervals of $0.3-1.1 \mu \mathrm{m}$ from each other. Small invaginations of the primary wall were present on the lateral aspect of the protrusions and in the spaces between protrusions. The layer of ground substance beneath the protrusions was $0.3-0.4 \mu \mathrm{m}$ in thickness; septa were evident within the cysts (Fig. 1c).

\section{Molecular analysis}

Genomic DNA was extracted from the 5 individual sarcocysts of $S$. wenzeli isolated from different chickens, and $18 S$ rDNA, $28 S$ rDNA, ITS1, cox 1 and $r p o B$ were successfully amplified using their DNA as templates. The five $18 S \mathrm{rDNA}$ sequences of $S$. wenzeli were $1747 \mathrm{bp}$ in length and shared an identity of $99.8-100 \%$ (average $99.8 \%$ identity). Therefore, only 4 sequences (GenBank: MT756990MT756993) were submitted to GenBank. The most similar sequence in GenBank was that of Sarcocystis sp. isolate Chicken-2016-DF-BR (MN845627) from chicken (99.9-100\% identity, average $99.9 \%$ identity), followed by $S$. anasi (EU553477) from mallard duck (Anas platyrhynchos) (99.8\% identity), S. albifronsi (EU502868) from white-fronted goose (Anser albifons) (99.7\% identity) and S. rileyi (KJ396583) from common eider (Somateria mollissima) (99.5\% identity).

The five $28 S$ rDNA sequences of $S$. wenzeli were 3279 bp in length and shared $99.7-100 \%$ identity (average 99.9\% identity). Therefore, only 4 sequences (GenBank: MT756986-MT756989) were deposited in GenBank. The most similar sequence in GenBank was that of S. albifronsi (EF079885) (99.3-99.1\% identity, average 99.2\% identity), followed by Sarcocystis sp. (MH898978) from Temminck's stint (Calidris temminckii) (98.9-99.2\% identity, average 99.1\% identity), S. anasi (EF079887) (99.0-99.2\% identity, average $99.1 \%$ identity), S. cornixi (EU553480) from hooded crow (Corvus cornix) (98.6-98.9\% identity, average $98.8 \%$ identity) and $S$. rileyi (GU188426) (98.3-98.5\% identity, average 98.4\% identity).

The five cox 1 sequences of $S$. wenzeli were $1142 \mathrm{bp}$ in length and were completely identical; therefore, only 1 sequence (MT761700) was submitted to GenBank. The most similar sequence in GenBank was that of Sarcocystis sp. isolate Chicken-2016-DF-BR (MN848337) from chicken (99.3\% identity), followed by $S$. albifronsi 


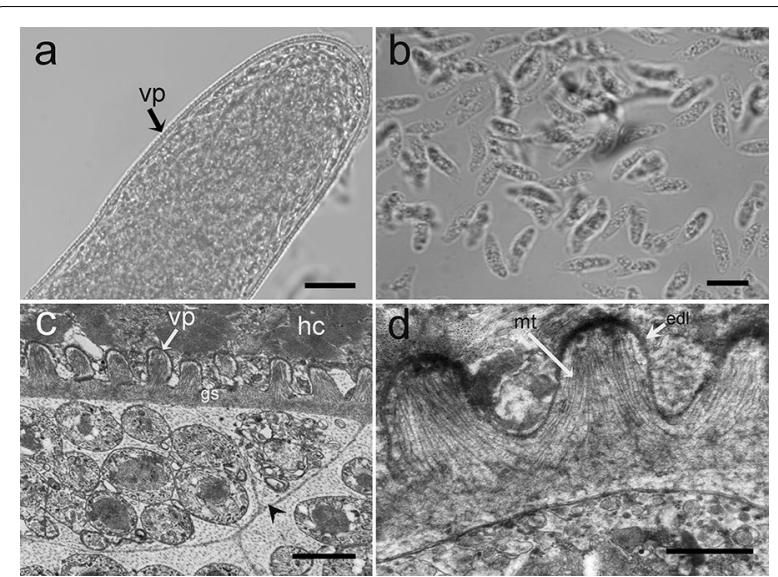

Fig. 1 Morphological characteristics of Sarcocystis wenzeli isolated from skeletal muscle in chickens. a LM micrograph of a sarcocyst (unstained). Note the short palisade-like villar protrusions (vp). b LM micrograph of bradyzoites (unstained). Note the lancet-shaped bradyzoites. c TEM micrograph of a sarcocyst. Note the stubby hill-like villar protrusions (vp), ground substance (gs), septa (arrowhead), and host cell (hc). d TEM micrograph of a sarcocyst. Note the electron-dense layer (edl) and the microtubules (mt). Scale-bars: $\mathbf{a}$ $20 \mu \mathrm{m} ; \mathbf{b}, 10 \mu \mathrm{m} ; \mathbf{c}, 2 \mu \mathrm{m} ; \mathbf{d}, 1 \mu \mathrm{m}$

(MH138310) (98.6\% identity), S. anasi (MH138311) (98.5\% identity) and S. rileyi (KJ396582) (96.4\% identity).

The five ITS1 sequences (MT756994-MT756998) of $S$. wenzeli were 1186-1187 bp in length and shared 99.0-99.9\% identity, with an average identity of $99.4 \%$. The most similar sequence was that of Sarcocystis sp. isolate Chicken-2016-DF-BR (MN846302) from chicken (98.1-98.5\% identity, average $98.3 \%$ identity), followed by S. anasi (JF520779) (89.3-89.7\% identity, average 89.5\% identity) and S. rileyi (KJ396584) (78.8-79.0\% identity, average $78.9 \%$ identity).

The five rpoB sequences of $S$. wenzeli were $844 \mathrm{bp}$ in length and shared 98.9-100\% identity, with an average identity of $99.3 \%$, so only 2 sequences (MT761694 and MT761695) were submitted to GenBank. The most similar sequence in GenBank was that of $S$. anasi (MH138320) (97.5\% identity), followed by those of $S$. albifronsi (MH138319) (97.4\% identity) and S. rileyi (MF596308) (95.9\% identity).

Although the newly obtained $18 S$ rDNA, cox 1 and ITS1 sequences had the highest similarity with homologous sequences of Sarcocystis sp. isolate Chicken-2016-DFBR, only the ITS1 sequence of this isolate was included in the phylogenetic analysis because sequences of the other two loci of the isolate were shorter than the rest and it would lower the phylogenetic signal of the analysis. In the phylogenetic tree constructed based on $18 \mathrm{~S}$ rDNA (Fig. 2), cox1 (Fig. 3), or rpoB (Fig. 4) sequences, S. wenzeli formed an individual clade with $S$. anasi, S. albifronsi and S. rileyi basal to a group comprising Sarcocysits spp. obtained from birds or terrestrial carnivores. Phylogenetic analysis based on ITS1 sequences (Fig. 5) revealed that $S$. wenzeli formed an individual clade with Sarcocysits sp. isolate Chicken-2016-DF-BR and S. rileyi, and this clade was within a group comprising Sarcocysits spp. obtained from birds or terrestrial carnivores.

\section{Discussion}

Sarcocystis spp. in chickens may cause severe myositis [14] and occasionally neurological disease [8, 15]. Sarcocystis infection in chickens has been reported in Hungary [1], Bulgaria [2], Russia [16], Papua New Guinea [14], Australia [14], Germany [3], the Czech Republic [17], Azerbaijan [18], China [19], Iran [20] and Brazil [8]. Three species of Sarcocystis, S. horvathi, S. wenzeli and S. gallinarum, have been proposed to be responsible for the sarcocysts observed in muscle tissues of chickens. The sarcocysts found in chickens have been divided into two types based on the shape of the bradyzoites. Bananashaped sarcocysts are considered to be produced by $S$. horvathi, described in 1908, which is synonymous with $S$. gallinarum whereas lancet-shaped sarcocysts are attributed to S. wenzeli, described in 1982 [5]. The ultrastructure of the sarcocysts of $S$. wenzeli has been described in detail previously $[19,21]$ and is similar to the type $9 \mathrm{k}$ sarcocyst wall classified by Dubey et al. [5]. It is worth noting that morphologically similar sarcocysts have been observed in the lesser snow geese (Anser caerulescens) in Saskatchewan, although the species has not been named [22]. The fine structure of the sarcocysts of $S$. horvathi and $S$. gallinarum is still unclear. In our materials, only the sarcocysts of $S$. wenzeli were found and identified, based on the observation of lancet-shaped bradyzoites and the TEM analysis of sarcocysts. The $42.4 \%(14 / 33)$ prevalence rate of Sarcocystis identified in chickens was lower than the $94.78 \%$ (37/39) prevalence recently surveyed in Iran using the digestive method [20], but was higher than the $8.9 \%(17 / 191)$ prevalence based on microscopic detection reported in China in 2012 [19]. It needs to be stressed that only squash preparation was used to search for mature sarcocysts in tissues of chickens in the present study. Therefore, the prevalence rate of Sarcocystis surveyed in the village should be underestimated because of the low sensitivity of the method.

Nucleotide sequence analysis has proven to be a useful tool for delineating or identifying species of Sarcocystis from the same or different hosts, and different genetic markers have revealed different levels of intra- or interspecific sequence diversity $[6,7,12]$. There are only one $18 S$ rDNA sequence, one ITS1 sequence and one cox 1 sequence of Sarcocystis sp. obtained from brains of two chickens in Brazil currently available in GenBank. In the 


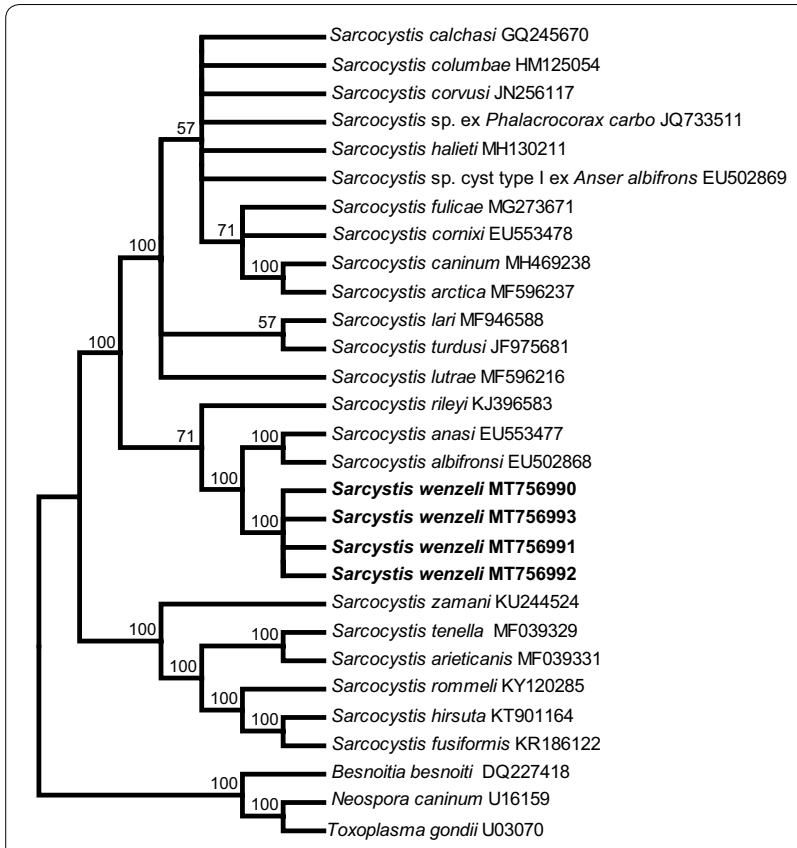

Fig. 2 Phylogenetic tree of selected members of the family Sarcocystidae based on 185 rDNA sequences, inferred using the maximum parsimony (MP) method with the tree bisection-regrafting algorithm (TBR). The values between the branches represent the percent bootstrap value per 1000 replicates, and values below 50\% are not shown. The GenBank accession numbers of all the sequences included in the analysis are given after the taxon names. The four new sequences of Sarcocystis wenzeli (MT756990-MT756993) are shown in boldface

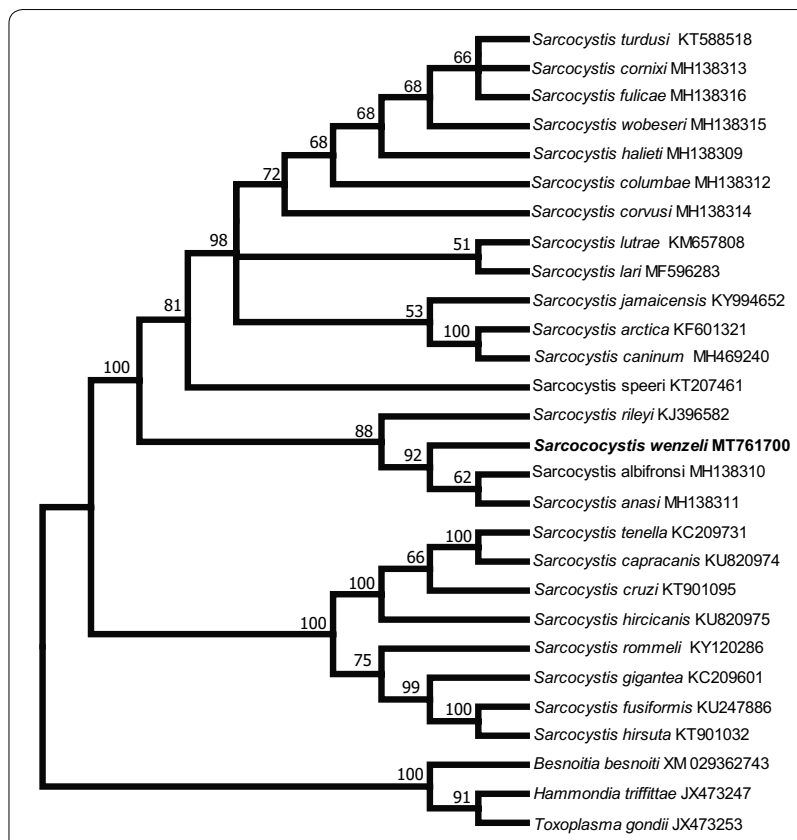

Fig. 3 Phylogenetic tree of selected members of the family Sarcocystidae based on mitochondrial cox 1 sequences, inferred using the maximum parsimony (MP) method with the tree bisection-regrafting algorithm (TBR). The values between the branches represent the percent bootstrap value per 1000 replicates, and values below $50 \%$ are not shown. The GenBank accession numbers of all the sequences included in the analysis are given after the taxon names. The new sequence of Sarcocystis wenzeli (MT761700) is shown in boldface present study, five loci (18S rDNA, 28S rDNA, ITS1, cox1 and $r p o B$ ) from $S$. wenzeli were sequenced and analyzed, to the best of our knowledge, for the first time. Among them, $28 S$ rDNA and rpoB constitutes the first records of Sarcocystis species in chickens. In our analysis, the sequences of the five loci (18S rDNA, $28 S$ rDNA, ITS1, $\operatorname{cox} 1$ and $r p o B$ ) of this parasite presented high intraspecific similarities of $99.8-100 \%, 99.7-100 \%, 99.0-99.9 \%$, $100 \%$, and $98.9-100 \%$, respectively. When comparing these sequences with those deposited on GenBank, sequences of $18 S$ rDNA, ITS1 and cox 1 of $S$. wenzeli shared high similarities with those of Sarcocystis sp. isolate Chicken-2016-DF-BR obtained from brains of two chickens, i.e. $99.9-100 \%, 98.1-98.5 \%$, and 99.3\% identity, respectively. Therefore, the unrecognized species of Sarcocystis associated with meningoencephalitis in chickens from Brazil in 2020 [8] could be inferred as S. wenzeli owing to the high similarities of the three loci. The first case of Sarcocysits-associated encephalitis in chickens was diagnosed in the USA in 1995 [15], and the species of Sarcocysits was not identified because of no sarcocysts observed in brain samples of chickens, similar to the case occurred in Brazil in 2020 [8]. The sequences of the five

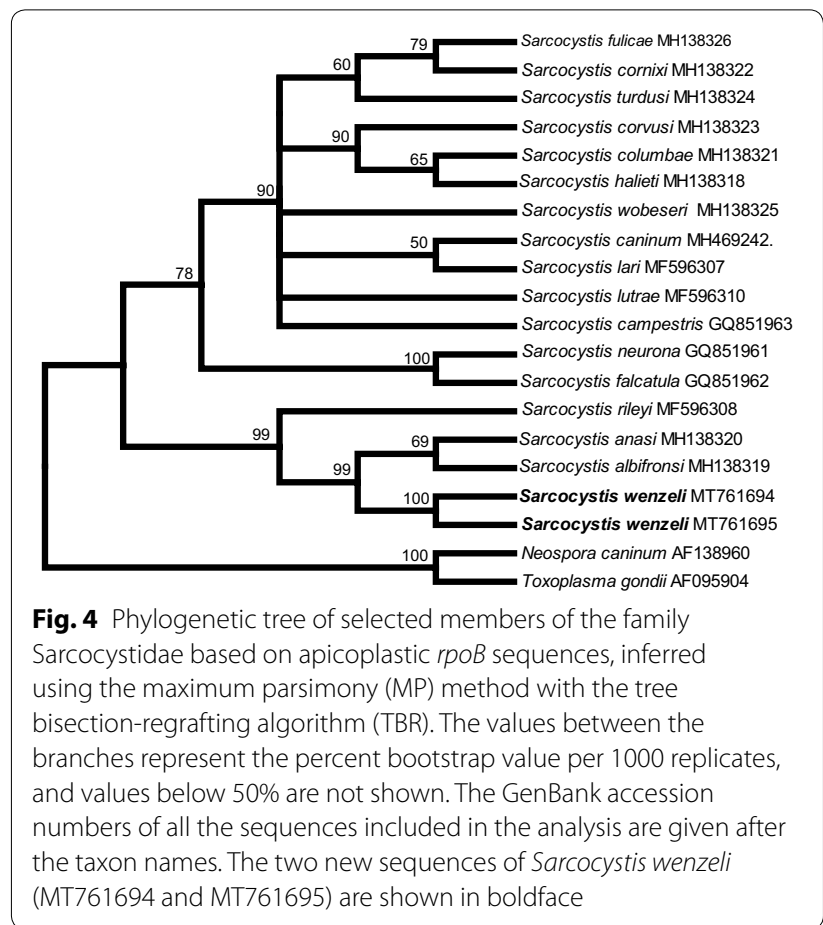




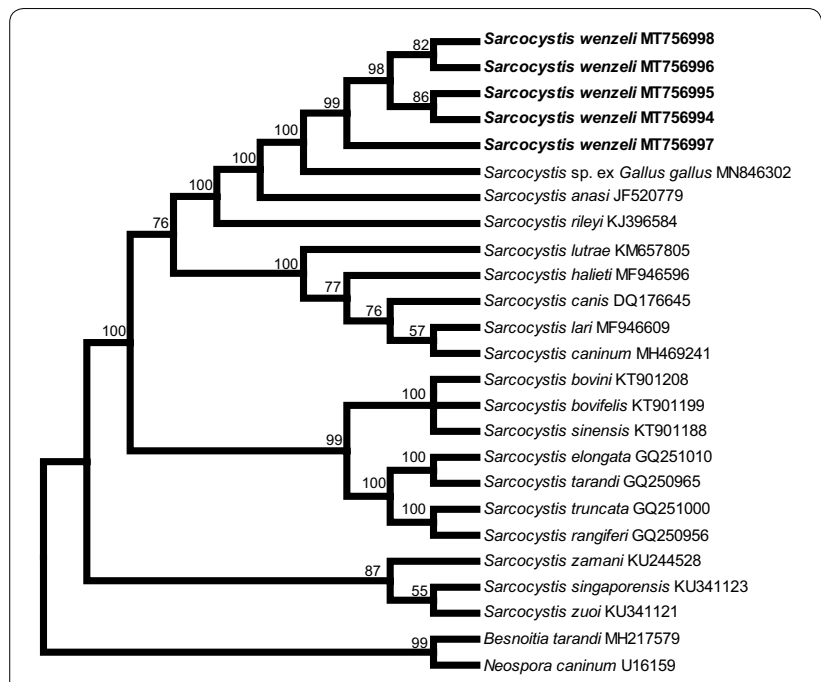

Fig. 5 Phylogenetic tree of selected members of the family Sarcocystidae based on ITS1 sequences, inferred using the maximum parsimony (MP) method with the tree bisection-regrafting algorithm (TBR). The values between the branches represent the percent bootstrap value per 1000 replicates. The GenBank accession numbers of all the sequences included in the analysis are given after the taxon names. The five new sequences of Sarcocystis wenzeli (MT756994-MT756998) are shown in boldface loci (18S rDNA, 28S rDNA, ITS1, cox1 and rpoB) of $S$. wenzeli exhibited different levels of similarity compared with closely related Sarcocystis species, sharing 99.8\%, 99.0-99.2\%, 89.3-89.7\%, 98.5\% and 97.5\% identity with the corresponding sequences of $S$. anasi, and 99.5\%, 98.3-98.5\%, 78.8-79.0\%, 96.4\%, and 95.9\% identity with those of S. rileyi. Therefore, ITS1 and rpoB appeared to be more suitable to for distinguishing $S$. wenzeli from other Sarcocystis spp., especially closely related species of Sarcocystis, than the $18 S$ rDNA, $28 S$ rDNA and cox 1 loci.

This study also established the phylogenetic relationships between S. wenzeli and Sarcocystis spp. in different hosts based on $18 S$ rDNA, cox1, rpoB and ITS1 sequences. The topologies of the trees inferred from these sequences were highly similar and revealed that $S$. wenzeli presents a close relationship with Sarcocystis sp. isolate Chicken-2016-DF-BR, S. rileyi, S. albifronsi and $S$. anasi. The later three species utilize geese or ducks as intermediate hosts, and the definitive hosts of $S$. rileyi and $S$. albifronsi are canines, but that of $S$. anasi is still unknown [23, 24]. Based on experimental infection, the definitive hosts of $S$. wenzeli were confirmed to be both cats and dogs $[3,25]$, which is peculiar and differs from the situation for all known Sarcocystis spp. found in domestic animals, which use only either cats or dogs as their definitive host. However, Sarcocystis sporocysts were not found in the feces of cats fed breast muscle sample from over 2000 chickens from grocery stores in the
USA, although the muscle was not examined microscopically for sarcocysts or bradyzoites [26].

\section{Conclusions}

In summary, we found a high prevalence rate of Sarcocystis in free-range chickens in China, and only S. wen$z e l i$ was identified based on the cyst ultrastructure. Five loci (18S rDNA, ITS1, $28 S$ rDNA, cox 1 and rpoB) of the parasite were sequenced, analyzed and deposited in GenBank. Based on molecular analysis, S. wenzeli might be an agent caused neurological disease in chickens. Among these genetic markers, ITS1 and rpoB are more suitable for discrimination among closely related Sarcocystis species. Phylogenetic analysis revealed that $S$. wenzeli shows a close relationship with Sarcocystis spp. that use geese and/or ducks as intermediate hosts and canines as definitive hosts.

\section{Abbreviations \\ LM: light microscopy; TEM: transmission electron microscopy; PCR: polymer- ase chain reaction; 185 rDNA: 185 ribosomal DNA; 285 rDNA: 285 ribosomal DNA; ITS1: intergenic transcribed spacer region 1; cox1: cytochrome oxidase subunit 1; rpoB: RNA polymerase beta subunit; MP: maximum parsimony; TBR: tree-bisection-regrafting.}

\section{Acknowledgements}

Not applicable.

\section{Authors' contributions}

$\mathrm{JH}$ conceived the study and prepared the manuscript. JP, CM and ZH collected the samples and performed the molecular experiments. $Y Y$ and $\mathrm{HZ}$ preformed the investigation of prevalence and morphological observation. SD and JT analyzed and interpreted the results. All authors read and approved the final manuscript

\section{Funding}

This study was supported by the National Key R\&D Program of China (grant 2017YFD0500400) and the Natural Sciences Foundation of China (Grant 31460557).

\section{Availability of data and materials}

The data collected for this study are available from the corresponding author upon request.

\section{Ethics approval and consent to participate}

The present study was approved by the Animal Ethics Committee of Yunnan University (permission number AEC2015021), and all authors declare that they have participated in this work.

\section{Consent for publication}

Not applicable.

\section{Competing interests}

The authors declare that they have no competing interests.

\section{Author details}

${ }^{1}$ School of Biological Sciences, Yunnan University, Kunming 650091, China. ${ }^{2}$ School of Ecology and Environmental Sciences and Yunnan Key Laboratory for Plateau Mountain Ecology and Restoration of Degraded Environments, Yunnan University, Kunming 650091, China. ${ }^{3}$ College of Veterinary Medicine, Yangzhou University, Yangzhou 225009, China. 
Received: 28 July 2020 Accepted: 6 October 2020

Published online: 14 October 2020

\section{References}

1. Rátz I. Szakosztályunk ülései Állattani Közlemények. 1908;7:177-8.

2. Krause C, Goranoff S. Ueber Sarkosporidiosis bei Huhn und Wildente. Z Infektionskr Haustier. 1933:43:261-78.

3. Wenzel R, Erber M, Boch J, Schellner HP. Sarkosporidien-Infektion be Haushuhn, Fasan und Bleßhuhn. Berl Münch Tierärztl Wochenschr. 1982;95:188-93.

4. Odening K. Die Sarcocystis-Infektion: Wechselbeziehungen zwischen freilebenden Wildtieren, Haustieren und Zootieren. Zool Garten N F. 1997;1997(67):317-40.

5. Dubey JP, Calero-Bernal R, Rosenthal BM, Speer CA, Fayer R. Sarcocystosis of animals and humans. 2nd ed. Boca Raton: CRC Press; 2016. p. 277-80.

6. Gjerde B. Phylogenetic relationships among Sarcocystis species in cervids, cattle and sheep inferred from the mitochondrial cytochrome c oxidase subunit I gene. Int J Parasitol. 2013;43:579-91.

7. Huang ZM, Ye YL, Zhang HZ, Deng SS, Tao JJ, Hu JJ, et al. Morphological and molecular characterizations of Sarcocystis miescheriana and Sarcocystis suihominis in domestic pigs (Sus scrofa) in China. Parasitol Res. 2019:118:3491-6.

8. Wilson TM, Sousa SKH, Paludo GR, de Melo CB, Llano HAB, Soares RM, et al. An undescribed species of Sarcocystis associated with necrotizing meningoencephalitis in naturally infected backyard chickens in the Midwest of Brazil. Parasitol Int. 2020;76:102098.

9. Fischer S, Odening K. Characterization of bovine Sarcocystis species by analysis of their 18S ribosomal DNA sequences. J Parasitol. 1998;84:50-4.

10. Kutkiené L, Prakas P, Sruoga A, Butkauskas D. The mallard duck (Anas platyrhynchos) as intermediate host for Sarcocystis wobeseri sp. nov. from the barnacle goose (Branta leucopsis). Parasitol Res. 2010;107:879-88.

11. Mugridge NB, Morrison DA, Heckeroth AR, Johnson AM, Tenter AM. Phylogenetic analysis based on full-length large subunit ribosomal RNA gene sequence comparison reveals that Neospora caninum is more closely related to Hammondia heydorni than to Toxoplasma gondii. Int J Parasitol. 1999;29:1545-56.

12. Hu JJ, Liu TT, Liu Q, Esch GW, Chen JQ, Huang S, et al. Prevalence, morphology, and molecular characteristics of Sarcocystis spp. in domestic goats (Capra hircus) from Kunming China. Parasitol Res. 2016:115:3973-81.

13. Kumar S, Stecher G, Li M, Knyaz C, Tamura K. MEGA X: Molecular Evolutionary Genetics Analysis across computing platforms. Mol Biol Evol. 2018;35:1547-9.
14. Munday BL, Humphrey JD, Kila V. Pathology produced by, prevalence of, and probable life-cycle of a species of Sarcocystis in the domestic fowl. Avian Dis. 1977:21:697-703.

15. Mutalib A, Keirs R, Maslin W, Topper M, Dubey JP. Sarcocystis-associated encephalitis in chickens. Avian Dis. 1995;39:436-40.

16. Golubkov IA. Infection of dogs and kittens with Sarcocystis from chickens and ducks. Veterinaria (Moscow). 1979;77:55-6

17. Pecka Z. Muscular sarcocystosis of fowls and pheasants in Czechoslovakia. Veterinářství. 1990;40:314-5.

18. Memmedov I. Prevalence of Sarcocystis species in some bird in Nakhchivan Autonomous Republic. Kafkas Univ Vet Fak Derg. 2010;16:857-60.

19. Chen X, He Y, Liu Y, Olias P, Rosenthal BM, Cui L, Zuo Y, Yang Z. Infections with Sarcocystis wenzeli are prevalent in the chickens of Yunnan Province, China, but not in the flocks of domesticated pigeons or ducks. Exp Parasitol. 2012;31:31-4

20. Vahedi Noori N, Salehi A, Razavi M, Masoumi M. Poultry carcasses investigation of parasitic Sarcocystis infection in native in Mazandaran (North part of Iran). Anim Husb Dairy Vet Sci. 2019;3:1-3.

21. Mehlhorn $\mathrm{H}$, Hartley WJ, Heydorn AO. A comparative ultrastructural study of the cyst wall of 13 Sarcocystis species. Protistologica. 1976;12:451-67.

22. Wobeser G, Leighton FA, Cawthorn RJ. Occurrence of Sarcocystis Lankester, 1882, in wild geese in Saskatchewan. Can J Zool. 1981;59:1621-4.

23. Kutkiené L, Prakas P, Sruoga A, Butkauskas D. Description of Sarcocystis anasi sp. nov. and Sarcocystis albifronsi sp. nov. in birds of the order Anseriformes. Parasitol Res. 2012;110:1043-6.

24. Prakas P, Liaugaudait S, Kutkienè L, Sruoga A, Švažas S. Molecular identification of Sarcocystis rileyi sporocysts in red foxes (Vulpes vulpes) and raccoon dogs (Nyctereutes procyonoides) in Lithuania. Parasitol Res. 2015;14:1671-6.

25. Mao JB, Zuo YX. Studies on the prevalence and experimental transmission of Sarcocystis sp. in chickens. Acta Vet Zootech Sinica. 1994:25:555-9.

26. Dubey JP, Hill DE, Jones JL, Hightower AW, Kirkland E, Roberts JM, et al. Prevalence of viable Toxoplasma gondii in beef, chicken, and pork from retail meat stores in the United States: risk assessment to consumers. J Parasitol. 2005;91:1082-93.

\section{Publisher's Note}

Springer Nature remains neutral with regard to jurisdictional claims in published maps and institutional affiliations. 